\title{
Risk of peanut- and tree-nut-induced anaphylaxis during Halloween, Easter and other cultural holidays in Canadian children
}

\author{
Mélanie Leung, Ann E. Clarke MD MSc, Sofianne Gabrielli MSc, Judy Morris MD MSc, Jocelyn Gravel MD MSc, \\ Rodrick Lim MD, Edmond S. Chan MD, Ran D. Goldman MD, Paul Enarson MD PhD, Andrew O'Keefe MD, \\ Jennifer Gerdts BComm, Derek Chu MD PhD, Julia Upton MD MPH, Xun Zhang PhD, Greg Shand MSc, \\ Moshe Ben-Shoshan MD MSc
}

Cite as: CMAJ 2020 September 21;192:E1084-92. doi: 10.1503/cmaj.200034

Visual abstract available at: https://www.cmaj.ca/lookup/doi/10.1503/cmaj.200034/tab-related-content

\begin{abstract}
BACKGROUND: It is not established whether the risk of anaphylaxis induced by peanuts or tree nuts in children increases at specific times of the year. We aimed to evaluate the risk of peanutand tree-nut-induced anaphylaxis during certain cultural holidays in Canadian children.
\end{abstract}

METHODS: We collected data on confirmed pediatric cases of anaphylaxis presenting to emergency departments in 4 Canadian provinces as part of the Cross-Canada Anaphylaxis Registry. We assessed the mean number of cases per day and incidence rate ratio (IRR) of anaphylaxis induced by unknown nuts, peanuts and tree nuts presenting during each of 6 holidays (Halloween, Christmas, Easter, Diwali, Chinese New Year and Eid al-Adha) versus the rest of the year. We estimated IRRs and 95\% confidence intervals $(\mathrm{Cls})$ using Poisson regression.

RESULTS: Data were collected for 1390 pediatric cases of anaphylaxis between 2011 and 2020. Their median age was 5.4 years, and 864 (62.2\%) of the children were boys. During Halloween and Easter, there were higher rates of anaphylaxis to unknown nuts (IRR 1.66, $95 \% \mathrm{Cl} 1.13-2.43$ and IRR 1.71, $95 \% \mathrm{Cl} 1.21-2.42$, respectively) and peanuts (IRR 1.86, 95\% Cl 1.12-3.11 and IRR $1.57,95 \% \mathrm{Cl} 0.94-2.63$, respectively) compared to the rest of the year. No increased risk of peanut- or tree-nutinduced anaphylaxis was observed during Christmas, Diwali, Chinese New Year or Eid al-Adha. Anaphylaxis induced by unknown nuts, peanuts and tree nuts was more likely in children aged 6 years or older than in younger children.

INTERPRETATION: We found an increased risk of anaphylaxis induced by unknown nuts and peanuts during $\mathrm{Hal}-$ loween and Easter among Canadian children. Educational tools are needed to increase awareness and vigilance in order to decrease the risk of anaphylaxis induced by peanuts and tree nuts in children during these holidays. n Canada, up to $9 \%$ of children have at least 1 food allergy. ${ }^{1-4}$ Anaphylaxis - an allergic reaction involving at least 2 organ systems or resulting in hypotension ${ }^{5}$ - is the most severe, lifethreatening manifestation of food allergy. We have reported that the incidence of anaphylaxis (mainly food-induced) presenting to the emergency department at 2 Canadian children's hospitals almost doubled from 2012 to 2016. , $^{6,7}$ Peanuts and tree nuts are the main culprits in food-induced anaphylaxis. ${ }^{4,8,9}$ Furthermore, peanuts and tree nuts account for most fatal cases of foodrelated anaphylaxis in North America. 8,10

Although specific periods in the year such as holidays are expected to be associated with a higher risk of accidental reactions to peanuts and tree nuts, to our knowledge, there are currently no data assessing this risk. Identifying certain times associated with an increased risk of anaphylaxis could help to raise community awareness, support and vigilance. This information would identify the best timing for public awareness campaigns to prevent allergic reactions. The primary objective of the current study was to assess the risk of peanut- and tree-nuttriggered anaphylaxis at Halloween, Christmas and Easter. We chose these holidays because they are probably the most celebrated holidays in Canada. The secondary objective was to determine factors that may be associated with an increased risk of such anaphylaxis during Diwali, Chinese New Year and Eid al-Adha. 


\section{Methods}

In 2011, we established a large registry that recruited patients with anaphylaxis presenting to pediatric emergency departments in 4 Canadian provinces: Quebec, Ontario, Newfoundland and Labrador, and British Columbia. The goal of the registry is to collect data on rates, triggers and management of anaphylaxis in different Canadian provinces.

In the current study, we included data from Apr. 15, 2011, to Jan. 31, 2020. We recruited patients younger than 18 years old who presented with unknown-nut, peanut- or tree-nut-induced anaphylaxis (Figure 1). We defined anaphylaxis as the involvement of at least 2 organ systems or hypotension in response to the culprit food. ${ }^{5}$ We collected data prospectively and retrospectively through a standardized data entry form. Prospective data were collected at the time of patient presentation. The treating emergency department physician along with a trained member of the research team identified cases of anaphylaxis ${ }^{5}$ and, after obtaining consent, completed the data entry form. We identified cases retrospectively through chart review to capture all patients presenting with International Statistical Classification of Diseases and Related Health Problems, 10th Revision codes related to anaphylaxis. These cases were then evaluated by 3 independent reviewers (S.G., M.B.-S., G.S.) to confirm that they fulfilled the definition of anaphylaxis, based on a previously validated algorithm. 5,9,11,12 This allowed capture of all cases that had been missed in prospective recruitment.

Using the standardized data entry form, we collected information on sociodemographic factors (age and sex), the date of presentation to the emergency department (which was the date of patient registration at the emergency department), the reported trigger food, a history of known peanut or tree nut allergy, the presence of comorbidities (e.g., asthma), clinical characteristics and management. We used 3 categories of reported trigger food for this study: unknown nut when the parents and treating physician were not sure whether the culprit was peanuts or tree nuts, and no other potential allergen had been consumed; peanut when the patient or parent attributed the anaphylactic reaction to peanuts only; and tree nut when tree nuts were the only identified culprit. We included the "unknown nut" category as it reflects real-life events.

To grade the severity of the anaphylactic reaction, we used a modified grading system elaborated by Muraro and colleagues. ${ }^{13,14}$ Mild reactions were defined as generalized pruritis, flushing, urticaria, angioedema, nausea or emesis, mild abdominal pain, nasal congestion or sneezing or both, rhinorrhea, throat tightness, mild wheezing, tachycardia or anxiety. Moderate reactions were defined as crampy abdominal pain, diarrhea, recurrent vomiting, hoarseness, "barky" cough, difficulty swallowing, dyspnea, moderate wheezing or "light-headedness." Severe reactions were those that manifested as a loss of bowel control, cyanosis, respiratory arrest, hypotension or circulatory collapse or both, dysrhythmia, severe bradycardia or cardiac arrest or both, confusion or loss of consciousness.

For the analysis, we included 3214 calendar days, from Apr. 15, 2011, to Jan. 31, 2020. The holiday time period was considered as the exposure in our study. Calendar days were units of observation and were classified into 4 multiday periods or categories for the primary analysis: Halloween, Christmas, Easter and the rest of the year (excluding the holidays). In secondary analyses, we extended our analysis to 7 categories: Halloween, Christmas, Easter, Diwali, Chinese New Year, Eid al-Adha and the

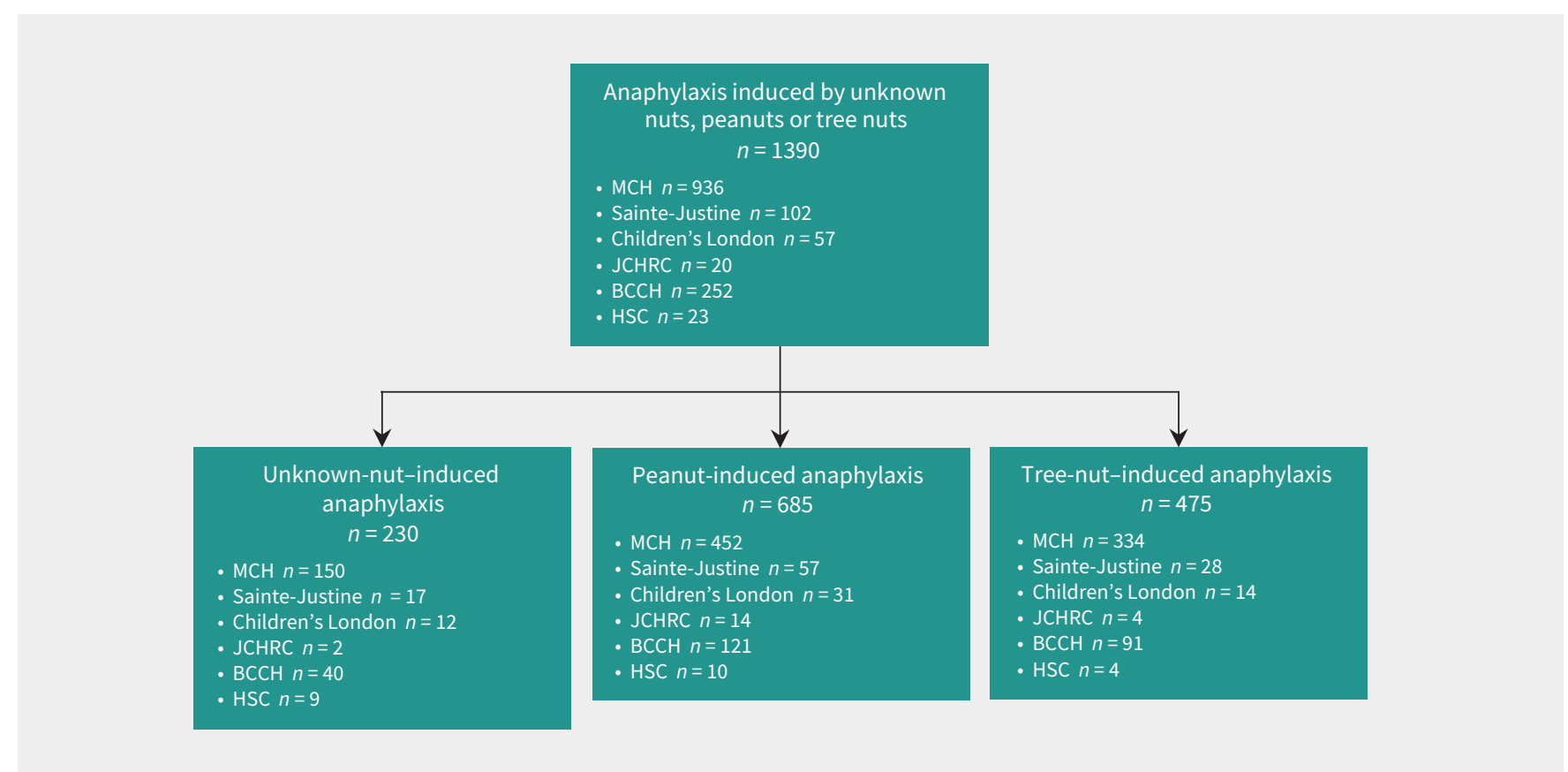

Figure 1: Patient recruitment sites. Note: BCCH = British Columbia Children's Hospital, Children's London = Children's Hospital at London Health Sciences Centre, HSC = Hôpital Sacré-Cœur (mainly for adults), JCHRC = Janeway Children's Health and Rehabilitation Centre, MCH = Montreal Children's Hospital, Sainte-Justine $=$ Centre hospitalier universitaire Sainte-Justine . 
rest of the year (excluding the holidays) (Box 1). We selected these holidays as they are some of the holidays most celebrated by children, and children are more likely to be exposed to peanuts and tree nuts during these times, given that they may be celebrated with extended family members or others handing out candies and other treats to children.

\section{Statistical analysis}

We used a Poisson regression model, based on observation from Apr. 15, 2011, to Jan. 31, 2020, to assess the changes in risk of peanut- and tree-nut-triggered anaphylaxis. We examined the mean number of cases per day of anaphylaxis triggered by unknown nuts, peanuts and tree nuts during Halloween, Christmas, Easter, Diwali, Chinese New Year and Eid al-Adha compared to the mean number of daily visits to the emergency department for peanut- or tree-nut-induced anaphyalxis during
Box 1: Dates of the holidays studied*

- Halloween: Oct. 31 of every year

- Christmas: Dec. 25 of every year

- Easter: first Sunday of Western Easter

- Diwali: 13th day of the seventh month in the Hindu calendar

- Chinese New Year: first day of the first month in the Chinese calendar

- Eid al-Adha: 10th day of the last month in the Islamic calendar

${ }^{*}$ Each holiday category was a 5-day period: the day preceding the holiday, the day of the celebration and the following 3 days. We expected the highest risk of exposure and reactions would be during this time interval, given that reactions may occur owing to early consumption and continuous consumption of foods associated with the holiday.

Table 1: Characteristics of patients who presented to the emergency department for anaphylaxis, by holiday

\begin{tabular}{|c|c|c|c|c|c|}
\hline \multirow[b]{2}{*}{ Characteristic } & \multicolumn{5}{|c|}{ No. $(\%)$ of cases*; holiday } \\
\hline & $\begin{array}{l}\text { Halloween } \\
\quad n=33\end{array}$ & $\begin{array}{c}\text { Christmas } \\
n=16\end{array}$ & $\begin{array}{l}\text { Easter } \\
n=33\end{array}$ & $\begin{array}{c}\text { Rest of year } \\
n=1308\end{array}$ & $\begin{array}{c}\text { Total } \\
n=1390\end{array}$ \\
\hline \multicolumn{6}{|l|}{ Sex } \\
\hline Male & $19(57.6)$ & $13(81.2)$ & $21(63.6)$ & $811(62.0)$ & $864(62.2)$ \\
\hline Female & $14(42.4)$ & $3(18.8)$ & $12(36.4)$ & $497(38.0)$ & $526(37.8)$ \\
\hline$p$ value $\dagger$ & 0.6 & 0.1 & 0.9 & Reference & NA \\
\hline Age, median (IQR), yr & $6.8(3.6-11.0)$ & $7.6(3.7-14.6)$ & $10.8(6.5-13.6)$ & $5.3(2.5-10.6)$ & $5.4(2.5-10.9)$ \\
\hline$p$ valuet & 0.48 & 0.1 & 0.002 & Reference & NA \\
\hline \multicolumn{6}{|l|}{ Recruitment method } \\
\hline Prospective & $13(39.4)$ & $2(12.5)$ & $20(60.6)$ & $553(42.3)$ & $588(42.3)$ \\
\hline Retrospective & $20(60.6)$ & $14(87.5)$ & $13(39.4)$ & $755(57.7)$ & $802(57.7)$ \\
\hline$p$ valuet & 0.7 & 0.02 & 0.03 & Reference & NA \\
\hline \multicolumn{6}{|l|}{ History of asthma } \\
\hline Yes & $6(18.2)$ & $4(25)$ & $8(24.2)$ & $213(16.3)$ & $231(16.6)$ \\
\hline No & $27(81.8)$ & $12(75)$ & $25(75.8)$ & $1088(83.2)$ & $1152(82.9)$ \\
\hline Information missing & $0(0.0)$ & $0(0.0)$ & $0(0.0)$ & $7(0.5)$ & $7(0.5)$ \\
\hline$p$ value $\dagger$ & 0.8 & 0.4 & 0.2 & Reference & NA \\
\hline \multicolumn{6}{|l|}{ Known peanut allergy } \\
\hline Yes & $14(42.4)$ & $5(31.3)$ & $12(36.4)$ & $498(38.1)$ & $529(38.1)$ \\
\hline No & $19(57.6)$ & $11(68.7)$ & $21(63.6)$ & $803(61.4)$ & $854(61.4)$ \\
\hline Information missing & $0(0.0)$ & $0(0.0)$ & $0(0.0)$ & $7(0.5)$ & $7(0.5)$ \\
\hline$p$ value $\dagger$ & 0.6 & 0.6 & 0.8 & Reference & NA \\
\hline \multicolumn{6}{|l|}{ Known tree nut allergy } \\
\hline Yes & $6(18.2)$ & $4(25)$ & $10(30.3)$ & $222(17.0)$ & $242(17.4)$ \\
\hline No & $27(81.8)$ & $12(75)$ & $23(69.7)$ & $1079(82.5)$ & $1141(82.1)$ \\
\hline Information missing & $0(0.0)$ & $0(0.0)$ & $0(0.0)$ & $7(0.5)$ & $7(0.5)$ \\
\hline$p$ valuet & 0.9 & 0.4 & 0.05 & Reference & NA \\
\hline
\end{tabular}


the rest of the year. We calculated and plotted the incidence rate ratio (IRR) for each holiday in comparison to the rest of the year. We also compared the daily number of cases between the week before and after each holiday. Furthermore, we performed a multivariable regression to evaluate the association between anaphylaxis on a given holiday and age, sex, and trigger food (peanuts or tree nuts).

We then stratified the Poisson regression by sociodemographic factors (sex, age), recruitment method (retrospective, prospective), presence of comorbidities (history of asthma), severity of reaction and hospital centre to evaluate whether these features modified the observed changes in risk of anaphylaxis induced by unknown nuts, peanuts and tree nuts during the holidays examined.

We analyzed all data using SAS version 9.4 (SAS Institute).

\section{Ethics approval}

The study was approved by the ethics committees of all participating hospitals: Montreal Children's Hospital; the Centre hospitalier universitaire Sainte-Justine, Montréal; the Children's Hospital at London Health Sciences Centre, London, Ont.; the Janeway Children's Health and Rehabilitation Centre, St. John's; British Columbia Children's Hospital, Vancouver; and Hôpital SacréCœur, Montréal.

\section{Results}

From 2011 to 2020, 1390 cases of peanut- or tree-nut-induced anaphylaxis were identified. Of the 1390 children involved, 864 $(62.2 \%)$ were boys; the median age was 5.4 years (interquartile range [IQR] 2.5-10.9 yr) (Table 1 ).

For anaphylaxis triggered by unknown nuts, during Halloween and Easter, there was an increase of about $70 \%$ in the average daily count compared to the rest of the year (IRR 1.66, $95 \% \mathrm{Cl} 1.13-2.43$ and IRR $1.71,95 \% \mathrm{Cl} 1.21-2.42$, respectively) (Table 2). The mean daily count was similar during Christmas, Diwali, Chinese New Year and Eid al-Adha compared to the rest of the year.

For peanut-triggered anaphylaxis, the mean daily count of events increased by $85 \%$ during Halloween compared to the rest of the year (IRR 1.86, 95\% Cl 1.12-3.11) (Table 2). During Easter, there was an increase of $60 \%$ compared to the rest of the year (IRR $1.57,95 \% \mathrm{Cl} 0.94-2.63$ ). No variation in the mean daily count of events was observed for the other holidays.

For anaphylaxis triggered by tree nuts, no variation in the mean daily count of events was observed for any holiday compared to the rest of the year (Table 2).

When we compared the mean daily count of events between the week before and after each holiday, no differences were found for any culprit food for any holiday (Appendix 1, Supplemental Tables S1 and S2, available at www.cmaj.ca/lookup/ suppl/doi:10.1503/cmaj.200034/-/DC1). In the multivariable analysis, apart from an association between age and tree nuts for Easter, we did not detect a significant association, possibly owing to the small sample (Appendix 1, Supplemental Table S3).

\section{Factors affecting the risk of anaphylaxis}

We conducted stratified analyses for Halloween, Christmas and Easter (Table 3, Table 4, Table 5). Given that no holiday effect was observed for Diwali, Chinese New Year and Eid al-Adha in the crude analysis, no stratified analysis was conducted.

Higher IRRs were observed for boys and girls for unknown nuts, and for boys for tree nuts during Halloween than during the rest of the year. Higher IRRs were identified for boys for unknown nuts, for boys and girls for peanuts, and for girls for tree nuts during Easter than during the rest of the year

\begin{tabular}{|c|c|c|c|c|c|c|c|}
\hline \multirow[b]{2}{*}{ Trigger food } & \multicolumn{7}{|c|}{ Holiday } \\
\hline & Rest of year & Halloween & Christmas & Easter & Diwali & $\begin{array}{c}\text { Chinese New } \\
\text { Year }\end{array}$ & Eid al-Adha \\
\hline \multicolumn{8}{|l|}{ Unknown nuts } \\
\hline $\begin{array}{l}\text { No. of cases per } \\
\text { day }(95 \% \mathrm{Cl})\end{array}$ & $0.43(0.40-0.45)$ & $0.71(0.49-1.04)$ & $0.36(0.22-0.58)$ & $0.73(0.52-1.03)$ & $0.47(0.30-0.72)$ & $0.25(0.13-0.46)$ & $0.42(0.27-0.66)$ \\
\hline IRR $(95 \% \mathrm{Cl})$ & Reference & $1.66(1.13-2.43)$ & $0.85(0.51-1.36)$ & $1.71(1.21-2.42)$ & $1.09(0.71-1.68)$ & $0.58(0.31-1.09)$ & $0.99(0.63-1.55)$ \\
\hline \multicolumn{8}{|l|}{ Peanuts } \\
\hline $\begin{array}{l}\text { No. of cases per } \\
\text { day }(95 \% \mathrm{CI})\end{array}$ & $0.21(0.20-0.23)$ & $0.39(0.24-0.65)$ & $0.13(0.06-0.30)$ & $0.33(0.20-0.55)$ & $0.24(0.14-0.44)$ & $0.13(0.05-0.30)$ & $0.16(0.07-0.33)$ \\
\hline IRR $(95 \% \mathrm{Cl})$ & Reference & $1.86(1.12-3.11)$ & $0.63(0.28-1.41)$ & $1.57(0.94-2.63)$ & $1.15(0.64-2.10)$ & $0.59(0.24-1.42)$ & $0.73(0.35-1.55)$ \\
\hline \multicolumn{8}{|l|}{ Tree nuts } \\
\hline $\begin{array}{l}\text { No. of cases per } \\
\text { day }(95 \% \mathrm{CI})\end{array}$ & $0.15(0.13-0.16)$ & $0.21(0.11-0.42)$ & $0.16(0.07-0.33)$ & $0.29(0.17-0.50)$ & $0.16(0.07-0.33)$ & $0.08(0.02-0.23)$ & $0.18(0.09-0.36)$ \\
\hline IRR (95\% CI) & Reference & $1.45(0.72-2.92)$ & $1.07(0.51-2.26)$ & $1.99(1.15-3.46)$ & $1.07(0.51-2.26)$ & $0.52(0.17-1.61)$ & $1.22(0.61-2.47)$ \\
\hline
\end{tabular}


Table 3: Stratified analysis of mean number of cases per day and incidence rate ratio for anaphylaxis induced by unknown nuts

\begin{tabular}{|c|c|c|c|c|c|c|c|}
\hline \multirow[b]{2}{*}{ Characteristic } & \multirow{2}{*}{$\begin{array}{c}\text { Rest of year; } \\
\text { no. of cases } \\
\text { per day } \\
(95 \% \mathrm{Cl})\end{array}$} & \multicolumn{2}{|c|}{ Halloween } & \multicolumn{2}{|c|}{ Christmas } & \multicolumn{2}{|c|}{ Easter } \\
\hline & & $\begin{array}{c}\text { No. of cases } \\
\text { per day } \\
(95 \% \mathrm{CI})\end{array}$ & $\begin{array}{c}\text { IRR } \\
(95 \% \mathrm{CI})^{\star}\end{array}$ & $\begin{array}{c}\text { No. of cases } \\
\text { per day } \\
(95 \% \mathrm{CI})\end{array}$ & $\begin{array}{c}\text { IRR } \\
(95 \% \mathrm{CI})^{\star}\end{array}$ & $\begin{array}{c}\text { No. of cases } \\
\text { per day } \\
(95 \% \mathrm{CI})\end{array}$ & $\begin{array}{c}\text { IRR } \\
(95 \% \mathrm{CI})^{*}\end{array}$ \\
\hline \multicolumn{8}{|l|}{ Sex } \\
\hline Female & $\begin{array}{c}0.16 \\
(0.15-0.18)\end{array}$ & $\begin{array}{c}0.31 \\
(0.18-0.53)\end{array}$ & $\begin{array}{c}1.93 \\
(1.13-3.28)\end{array}$ & $\begin{array}{c}0.07 \\
(0.02-0.21)\end{array}$ & $\begin{array}{c}0.41 \\
(0.13-1.28)\end{array}$ & $\begin{array}{c}0.27 \\
(0.15-0.47)\end{array}$ & $\begin{array}{c}1.65 \\
(0.93-2.93)\end{array}$ \\
\hline Male & $\begin{array}{c}0.26 \\
(0.25-0.28)\end{array}$ & $\begin{array}{c}0.42 \\
(0.27-0.66)\end{array}$ & $\begin{array}{c}1.60 \\
(1.02-2.53)\end{array}$ & $\begin{array}{c}0.29 \\
(0.17-0.50)\end{array}$ & $\begin{array}{c}1.10 \\
(0.63-1.90)\end{array}$ & $\begin{array}{c}0.47 \\
(0.30-0.72)\end{array}$ & $\begin{array}{c}1.77 \\
(1.15-2.73)\end{array}$ \\
\hline \multicolumn{8}{|l|}{ Age, yr } \\
\hline$<6$ & $\begin{array}{c}0.23 \\
(0.21-0.25)\end{array}$ & $\begin{array}{c}0.29 \\
(0.17-0.50)\end{array}$ & $\begin{array}{c}1.26 \\
(0.73-2.18)\end{array}$ & $\begin{array}{c}0.13 \\
(0.06-0.30)\end{array}$ & $\begin{array}{c}0.58 \\
(0.26-1.30)\end{array}$ & $\begin{array}{c}0.18 \\
(0.09-0.36)\end{array}$ & $\begin{array}{c}0.78 \\
(0.39-1.56)\end{array}$ \\
\hline$\geq 6$ & $\begin{array}{c}0.20 \\
(0.18-0.21)\end{array}$ & $\begin{array}{c}0.44 \\
(0.29-0.69)\end{array}$ & $\begin{array}{c}2.27 \\
(1.46-3.55)\end{array}$ & $\begin{array}{c}0.22 \\
(0.12-0.41)\end{array}$ & $\begin{array}{c}1.14 \\
(0.61-2.12)\end{array}$ & $\begin{array}{c}0.56 \\
(0.38-0.82)\end{array}$ & $\begin{array}{c}2.84 \\
(1.90-4.24)\end{array}$ \\
\hline \multicolumn{8}{|c|}{ Recruitment method } \\
\hline Retrospective & $\begin{array}{c}0.25 \\
(0.23-0.26)\end{array}$ & $\begin{array}{c}0.44 \\
(0.29-0.69)\end{array}$ & $\begin{array}{c}1.81 \\
(1.16-2.83)\end{array}$ & $\begin{array}{c}0.31 \\
(0.18-0.53)\end{array}$ & $\begin{array}{c}1.27 \\
(0.75-2.15)\end{array}$ & $\begin{array}{c}0.29 \\
(0.17-0.50)\end{array}$ & $\begin{array}{c}1.18 \\
(0.68-2.04)\end{array}$ \\
\hline Prospective & $\begin{array}{c}0.18 \\
(0.17-0.20)\end{array}$ & $\begin{array}{c}0.29 \\
(0.17-0.50)\end{array}$ & $\begin{array}{c}1.61 \\
(0.93-2.79)\end{array}$ & $\begin{array}{c}0.04 \\
(0.01-0.18)\end{array}$ & $\begin{array}{c}0.25 \\
(0.06-0.99)\end{array}$ & $\begin{array}{c}0.44 \\
(0.29-0.69)\end{array}$ & $\begin{array}{c}2.47 \\
(1.58-3.87)\end{array}$ \\
\hline \multicolumn{8}{|l|}{ History of asthma } \\
\hline Yes & $\begin{array}{c}0.07 \\
(0.06-0.08)\end{array}$ & $\begin{array}{c}0.13 \\
(0.06-0.30)\end{array}$ & $\begin{array}{c}1.93 \\
(0.86-4.34)\end{array}$ & $\begin{array}{c}0.09 \\
(0.03-0.24)\end{array}$ & $\begin{array}{c}1.28 \\
(0.48-3.46)\end{array}$ & $\begin{array}{c}0.18 \\
(0.09-0.36)\end{array}$ & $\begin{array}{c}2.57 \\
(1.27-5.21)\end{array}$ \\
\hline No & $\begin{array}{c}0.35 \\
(0.33-0.38)\end{array}$ & $\begin{array}{c}0.60 \\
(0.41-0.87)\end{array}$ & $\begin{array}{c}1.70 \\
(1.16-2.49)\end{array}$ & $\begin{array}{c}0.27 \\
(0.15-0.47)\end{array}$ & $\begin{array}{c}0.75 \\
(0.43-1.33)\end{array}$ & $\begin{array}{c}0.55 \\
(0.38-0.82)\end{array}$ & $\begin{array}{c}1.57 \\
(1.06-2.34)\end{array}$ \\
\hline \multicolumn{8}{|c|}{ Severity of reaction } \\
\hline Mild & $\begin{array}{c}0.09 \\
(0.08-0.10)\end{array}$ & $\begin{array}{c}0.11 \\
(0.05-0.27)\end{array}$ & $\begin{array}{c}1.31 \\
(0.54-3.16)\end{array}$ & $\begin{array}{c}0.11 \\
(0.05-0.27)\end{array}$ & $\begin{array}{c}1.31 \\
(0.54-3.16)\end{array}$ & $\begin{array}{c}0.09 \\
(0.03-0.24)\end{array}$ & $\begin{array}{c}1.04 \\
(0.39-2.80)\end{array}$ \\
\hline Moderate & $\begin{array}{c}0.32 \\
(30-0.34)\end{array}$ & $\begin{array}{c}0.60 \\
(0.41-0.87)\end{array}$ & $\begin{array}{c}1.90 \\
(1.29-2.78)\end{array}$ & $\begin{array}{c}0.22 \\
(0.12-0.41)\end{array}$ & $\begin{array}{c}0.70 \\
(0.38-1.31)\end{array}$ & $\begin{array}{c}0.58 \\
(0.39-0.85)\end{array}$ & $\begin{array}{c}1.83 \\
(1.24-2.70)\end{array}$ \\
\hline Severe & $\begin{array}{c}0.02 \\
(0.02-0.03)\end{array}$ & $\begin{array}{c}0.02 \\
(0.00-0.16)\end{array}$ & $\begin{array}{c}0.96 \\
(0.13-6.94)\end{array}$ & $\begin{array}{c}0.02 \\
(0.00-0.16)\end{array}$ & $\begin{array}{c}0.96 \\
(0.13-6.94)\end{array}$ & $\begin{array}{c}0.07 \\
(9.02-0.21)\end{array}$ & $\begin{array}{c}2.89 \\
(0.91-9.18)\end{array}$ \\
\hline \multicolumn{8}{|l|}{ Hospital centre } \\
\hline $\mathrm{MCH}$ & $\begin{array}{c}0.29 \\
(0.27-0.30)\end{array}$ & $\begin{array}{c}0.56 \\
(0.38-0.82)\end{array}$ & $\begin{array}{c}1.95 \\
(1.31-2.90)\end{array}$ & $\begin{array}{c}0.31 \\
(0.18-0.53)\end{array}$ & $\begin{array}{c}1.09 \\
(0.64-1.85)\end{array}$ & $\begin{array}{c}0.42 \\
(0.27-0.66)\end{array}$ & $\begin{array}{c}1.48 \\
(0.94-2.33)\end{array}$ \\
\hline $\mathrm{BCCH}$ & $\begin{array}{c}0.08 \\
(0.07-0.09)\end{array}$ & $\begin{array}{c}0.11 \\
(0.05-0.27)\end{array}$ & $\begin{array}{c}1.44 \\
(0.60-3.50)\end{array}$ & $\begin{array}{c}0.04 \\
(0.01-0.18)\end{array}$ & $\begin{array}{c}0.58 \\
(0.14-2.32)\end{array}$ & $\begin{array}{c}0.18 \\
(0.09-0.36)\end{array}$ & $\begin{array}{c}2.31 \\
(1.14-4.67)\end{array}$ \\
\hline
\end{tabular}

Note: $\mathrm{BCCH}=$ British Columbia Children's Hospital, $\mathrm{Cl}=$ confidence interval, IRR = incidence rate ratio, $\mathrm{MCH}=$ Montreal Children's Hospital

${ }^{*}$ Calculated with rest of year as reference category.

For our age-stratified analysis, we used a cut-off age of 6 years given that, in Canada, most children are in their first year of elementary school at that age. Among children aged 6 years or older, we observed higher rates of anaphylaxis triggered by unknown nuts and peanuts during Halloween and Easter than during the rest of the year. Holidays did not affect the IRRs for children aged younger than 6 years for all 3 categories of trigger food.

Neither the presence of asthma nor the reaction severity were associated with the IRRs during holidays.

Children who were recruited prospectively had a higher IRR during Easter than during the rest of the year for anaphyalxis induced by unknown nuts, peanuts and tree nuts. However, for children recruited retrospectively, there was a higher IRR during Halloween than during the rest of the year for unknownnut- and peanut-induced anaphylaxis. Patient sociodemographic and clinical characteristics did not differ between the 2 groups (Table 6 ).

Our analysis stratified by hospital centre included only the Montreal Children's Hospital and British Columbia Children's Hospital, as they were the only sites with sufficient sample sizes. The IRRs for anaphylaxis induced by unknown nuts and peanuts were higher during Halloween than during the rest of 
Table 4: Stratified analysis of mean number of cases per day and incidence rate ratio for peanut-induced anaphylaxis

\begin{tabular}{|c|c|c|c|c|c|c|c|}
\hline \multirow[b]{2}{*}{ Characteristic } & \multirow{2}{*}{$\begin{array}{c}\text { Rest of year; } \\
\text { no. of cases } \\
\text { per day } \\
(95 \% \mathrm{Cl})\end{array}$} & \multicolumn{2}{|c|}{ Halloween } & \multicolumn{2}{|c|}{ Christmas } & \multicolumn{2}{|c|}{ Easter } \\
\hline & & $\begin{array}{c}\text { No. of cases } \\
\text { per day } \\
(95 \% \mathrm{Cl})\end{array}$ & $\begin{array}{c}\text { IRR } \\
(95 \% \mathrm{CI})^{*}\end{array}$ & $\begin{array}{c}\text { No. of cases } \\
\text { per day } \\
(95 \% \mathrm{Cl})\end{array}$ & $\begin{array}{c}\text { IRR } \\
(95 \% \mathrm{CI})^{*}\end{array}$ & $\begin{array}{c}\text { No. of cases } \\
\text { per day } \\
(95 \% \mathrm{Cl})\end{array}$ & $\begin{array}{c}\text { IRR } \\
(95 \% \mathrm{CI})^{*}\end{array}$ \\
\hline \multicolumn{8}{|l|}{ Sex } \\
\hline Female & $\begin{array}{c}0.08 \\
(0.07-0.09)\end{array}$ & $\begin{array}{c}0.16 \\
(0.07-0.33)\end{array}$ & $\begin{array}{c}1.92 \\
(0.91-4.08)\end{array}$ & $\begin{array}{c}0.04 \\
(0.01-0.18)\end{array}$ & $\begin{array}{c}0.55 \\
(0.14-2.21)\end{array}$ & $\begin{array}{c}0.09 \\
(0.03-0.24)\end{array}$ & $\begin{array}{c}1.10 \\
(0.41-2.95)\end{array}$ \\
\hline Male & $\begin{array}{c}0.13 \\
(0.12-0.14)\end{array}$ & $\begin{array}{c}0.24 \\
(0.14-0.44)\end{array}$ & $\begin{array}{c}1.90 \\
(1.04-3.45)\end{array}$ & $\begin{array}{c}0.09 \\
(0.03-0.24)\end{array}$ & $\begin{array}{c}0.69 \\
(0.26-1.85)\end{array}$ & $\begin{array}{c}0.24 \\
(0.14-0.44)\end{array}$ & $\begin{array}{c}1.90 \\
(1.04-3.45)\end{array}$ \\
\hline \multicolumn{8}{|l|}{ Age, yr } \\
\hline$<6$ & $\begin{array}{c}0.12 \\
(0.11-0.13)\end{array}$ & $\begin{array}{c}0.18 \\
(0.09-0.36)\end{array}$ & $\begin{array}{c}1.52 \\
(0.75-3.06)\end{array}$ & $\begin{array}{c}0.02 \\
(0.00-0.16)\end{array}$ & $\begin{array}{c}0.19 \\
(0.03-1.35)\end{array}$ & $\begin{array}{c}0.09 \\
(0.03-0.24)\end{array}$ & $\begin{array}{c}0.76 \\
(0.28-2.03)\end{array}$ \\
\hline$\geq 6$ & $\begin{array}{c}0.09 \\
(0.08-0.10)\end{array}$ & $\begin{array}{c}0.22 \\
(0.12-0.41)\end{array}$ & $\begin{array}{c}2.40 \\
(1.28-4.51)\end{array}$ & $\begin{array}{c}0.11 \\
(0.05-0.27)\end{array}$ & $\begin{array}{c}1.20 \\
(0.50-2.91)\end{array}$ & $\begin{array}{c}0.24 \\
(0.14-0.44)\end{array}$ & $\begin{array}{c}2.64 \\
(1.45-4.82)\end{array}$ \\
\hline \multicolumn{8}{|c|}{ Recruitment method } \\
\hline Retrospective & $\begin{array}{c}0.12 \\
(0.11-0.13)\end{array}$ & $\begin{array}{c}0.27 \\
(0.15-0.47)\end{array}$ & $\begin{array}{c}2.26 \\
(1.27-4.02)\end{array}$ & $\begin{array}{c}0.13 \\
(0.06-0.30)\end{array}$ & $\begin{array}{c}1.13 \\
(0.50-2.53)\end{array}$ & $\begin{array}{c}0.09 \\
(0.03-0.24)\end{array}$ & $\begin{array}{c}0.75 \\
(0.28-2.02)\end{array}$ \\
\hline Prospective & $\begin{array}{c}0.09 \\
(0.08-0.10)\end{array}$ & $\begin{array}{c}0.13 \\
(0.06-0.30)\end{array}$ & $\begin{array}{c}1.45 \\
(0.65-3.26)\end{array}$ & $0.0 \dagger \ddagger$ & $0.0 \ddagger$ & $\begin{array}{c}0.24 \\
(0.14-0.44)\end{array}$ & $\begin{array}{c}2.66 \\
(1.46-4.86)\end{array}$ \\
\hline \multicolumn{8}{|l|}{ History of asthma } \\
\hline Yes & $\begin{array}{c}0.03 \\
(0.03-0.04)\end{array}$ & $\begin{array}{c}0.09 \\
(0.03-0.34)\end{array}$ & $\begin{array}{c}2.56 \\
(0.94-6.94)\end{array}$ & $\begin{array}{c}0.04 \\
(0.01-0.18)\end{array}$ & $\begin{array}{c}1.28 \\
(0.32-5.18)\end{array}$ & $\begin{array}{c}0.09 \\
(0.03-0.34)\end{array}$ & $\begin{array}{c}2.56 \\
(0.94-6.94)\end{array}$ \\
\hline No & $\begin{array}{c}0.17 \\
(0.16-0.19)\end{array}$ & $\begin{array}{c}0.31 \\
(0.18-0.53)\end{array}$ & $\begin{array}{c}1.79 \\
(1.06-3.05)\end{array}$ & $\begin{array}{c}0.09 \\
(0.03-0.24)\end{array}$ & $\begin{array}{c}0.51 \\
(0.19-0.37)\end{array}$ & $\begin{array}{c}0.24 \\
(0.14-0.44)\end{array}$ & $\begin{array}{c}1.41 \\
(0.78-2.56)\end{array}$ \\
\hline \multicolumn{8}{|c|}{ Severity of reaction } \\
\hline Mild & $\begin{array}{c}0.04 \\
(0.04-0.05)\end{array}$ & $\begin{array}{c}0.09 \\
(0.03-0.24)\end{array}$ & $\begin{array}{c}2.06 \\
(0.76-5.56)\end{array}$ & $\begin{array}{c}0.02 \\
(0.00-0.16)\end{array}$ & $\begin{array}{c}0.51 \\
(0.07-3.68)\end{array}$ & $0.0 \dagger \ddagger$ & $0.0 \ddagger$ \\
\hline Moderate & $\begin{array}{c}0.15 \\
(0.14-0.17)\end{array}$ & $\begin{array}{c}0.29 \\
(0.17-0.50)\end{array}$ & $\begin{array}{c}1.87 \\
(1.08-3.24)\end{array}$ & $\begin{array}{c}0.09 \\
(0.03-0.24)\end{array}$ & $\begin{array}{c}0.57 \\
(0.21-1.54)\end{array}$ & $\begin{array}{c}0.29 \\
(0.17-0.50)\end{array}$ & $\begin{array}{c}1.87 \\
(1.08-3.24)\end{array}$ \\
\hline Severe & $\begin{array}{c}0.01 \\
(0.01-0.2)\end{array}$ & $\begin{array}{c}0.02 \\
(0.00-0.16)\end{array}$ & $\begin{array}{c}1.85 \\
(0.25-13.48)\end{array}$ & $\begin{array}{c}0.02 \\
(0.00-0.16)\end{array}$ & $\begin{array}{c}1.85 \\
(0.25-13.48)\end{array}$ & $\begin{array}{c}0.04 \\
(0.01-0.18)\end{array}$ & $\begin{array}{c}3.70 \\
(0.89-15.35)\end{array}$ \\
\hline \multicolumn{8}{|l|}{ Hospital centre } \\
\hline $\mathrm{MCH}$ & $\begin{array}{c}0.14 \\
(0.13-0.15)\end{array}$ & $\begin{array}{c}0.33 \\
(0.20-0.55)\end{array}$ & $\begin{array}{c}2.41 \\
(1.44-4.04)\end{array}$ & $\begin{array}{c}0.11 \\
(0.05-0.27)\end{array}$ & $\begin{array}{c}0.80 \\
(0.33-1.94)\end{array}$ & $\begin{array}{c}0.16 \\
(0.07-0.33)\end{array}$ & $\begin{array}{c}1.13 \\
(0.53-2.38)\end{array}$ \\
\hline $\mathrm{BCCH}$ & $\begin{array}{c}0.04 \\
(0.03-0.05)\end{array}$ & $\begin{array}{c}0.02 \\
(0.00-0.16)\end{array}$ & $\begin{array}{c}0.58 \\
(0.08-4.19)\end{array}$ & $\begin{array}{c}0.02 \\
(0.00-0.16)\end{array}$ & $\begin{array}{c}0.58 \\
(0.08-4.19)\end{array}$ & $\begin{array}{c}0.04 \\
(0.01-0.18)\end{array}$ & $\begin{array}{c}1.17 \\
(0.29-4.73)\end{array}$ \\
\hline
\end{tabular}

the year at the Montreal Children's Hospital but were higher during Easter than during the rest of the year at British Columbia Children's Hospital.

\section{Interpretation}

We found a higher risk of anaphylaxis induced by unknown nuts and peanuts during Halloween and Easter than during the rest of the year in Canadian children. No increased risk was observed during Christmas, Diwali, Chinese New Year or Eid alAdha. The difference in the anaphylaxis incidence among holidays may have been due to the social setting in which each holiday takes place. At Halloween and Easter, children often receive candies and other treats from people who may be unaware of their allergies. The absence of such an association at Christmas may be because Christmas is a more intimate celebration among family members and close friends, who are more vigilant regarding allergen exposure. ${ }^{15-17}$ Canadian labelling regulations may also contribute to our findings. Prepackaged 1-bite snacks and candies are commonly distributed on Halloween and Easter. These snacks, if sold individually, are exempt from labelling requirements, such as labelling of common allergens. ${ }^{18} \mathrm{We}$ found that the majority of anaphylactic reactions to tree nuts (in contrast to peanuts) occurred in children not known to have a tree nut allergy. This observation highlights the need for vigilance regarding first exposures to tree nuts during holidays. 
Table 5: Stratified analysis of mean number of cases per day and incidence rate ratio for anaphylaxis induced by tree nuts

\begin{tabular}{|c|c|c|c|c|c|c|c|}
\hline \multirow[b]{2}{*}{ Characteristic } & \multirow{2}{*}{$\begin{array}{c}\text { Rest of year; } \\
\text { no. of cases } \\
\text { per day } \\
(95 \% \mathrm{Cl})\end{array}$} & \multicolumn{2}{|c|}{ Halloween } & \multicolumn{2}{|c|}{ Christmas } & \multicolumn{2}{|c|}{ Easter } \\
\hline & & $\begin{array}{c}\text { No. of cases } \\
\text { per day } \\
(95 \% \mathrm{CI})\end{array}$ & $\begin{array}{c}\text { IRR } \\
(95 \% \mathrm{CI})^{*}\end{array}$ & $\begin{array}{c}\text { No. of cases } \\
\text { per day } \\
(95 \% \mathrm{CI})\end{array}$ & $\begin{array}{c}\text { IRR } \\
(95 \% \mathrm{CI})^{*}\end{array}$ & $\begin{array}{c}\text { No. of cases } \\
\text { per day } \\
(95 \% \mathrm{CI})\end{array}$ & $\begin{array}{c}\text { IRR } \\
(95 \% \mathrm{CI})^{*}\end{array}$ \\
\hline \multicolumn{8}{|l|}{ Sex } \\
\hline Female & $\begin{array}{c}0.05 \\
(0.04-0.06)\end{array}$ & $\begin{array}{c}0.13 \\
(0.06-0.30)\end{array}$ & $\begin{array}{c}2.72 \\
(1.20-6.15)\end{array}$ & $0.0 \dagger \ddagger$ & $0.0 \ddagger$ & $\begin{array}{c}0.13 \\
(0.06-0.30)\end{array}$ & $\begin{array}{c}2.72 \\
(1.20-6.15)\end{array}$ \\
\hline Male & $\begin{array}{c}0.10 \\
(0.09-0.11)\end{array}$ & $\begin{array}{c}0.07 \\
(0.02-0.21)\end{array}$ & $\begin{array}{c}0.70 \\
(0.22-2.17)\end{array}$ & $\begin{array}{c}0.16 \\
(0.07-0.33)\end{array}$ & $\begin{array}{c}1.62 \\
(0.77-3.44)\end{array}$ & $\begin{array}{c}0.16 \\
(0.07-0.33)\end{array}$ & $\begin{array}{c}1.62 \\
(0.77-3.44)\end{array}$ \\
\hline \multicolumn{8}{|l|}{ Age, yr } \\
\hline$<6$ & $\begin{array}{c}0.08 \\
(0.07-0.09)\end{array}$ & $\begin{array}{c}0.09 \\
(0.03-0.24)\end{array}$ & $\begin{array}{c}1.13 \\
(0.42-3.04)\end{array}$ & $\begin{array}{c}0.09 \\
(0.03-0.24)\end{array}$ & $\begin{array}{c}1.13 \\
(0.42-3.04)\end{array}$ & $\begin{array}{c}0.07 \\
(0.02-0.21)\end{array}$ & $\begin{array}{c}0.85 \\
(0.27-2.65)\end{array}$ \\
\hline$\geq 6$ & $\begin{array}{c}0.07 \\
(0.06-0.08)\end{array}$ & $\begin{array}{c}0.11 \\
(0.05-0.27)\end{array}$ & $\begin{array}{c}1.68 \\
(0.69-4.07)\end{array}$ & $\begin{array}{c}0.07 \\
(0.02-0.21)\end{array}$ & $\begin{array}{c}1.01 \\
(0.32-3.15)\end{array}$ & $\begin{array}{c}0.22 \\
(0.12-0.41)\end{array}$ & $\begin{array}{c}3.35 \\
(1.78-6.33)\end{array}$ \\
\hline \multicolumn{8}{|c|}{ Recruitment method } \\
\hline Retrospective & $\begin{array}{c}0.09 \\
(0.08-0.10)\end{array}$ & $\begin{array}{c}0.07 \\
(0.02-0.21)\end{array}$ & $\begin{array}{c}0.77 \\
(0.25-2.39)\end{array}$ & $\begin{array}{c}0.11 \\
(0.05-0.27)\end{array}$ & $\begin{array}{c}1.28 \\
(0.53-3.09)\end{array}$ & $\begin{array}{c}0.16 \\
(0.07-0.33)\end{array}$ & $\begin{array}{c}1.79 \\
(0.84-3.78)\end{array}$ \\
\hline Prospective & $\begin{array}{c}0.06 \\
(0.05-0.07)\end{array}$ & $\begin{array}{c}0.13 \\
(0.06-0.30)\end{array}$ & $\begin{array}{c}2.31 \\
(1.02-5.20)\end{array}$ & $\begin{array}{c}0.04 \\
(0.01-0.18)\end{array}$ & $\begin{array}{c}0.77 \\
(0.19-3.10)\end{array}$ & $\begin{array}{c}0.13 \\
(0.06-0.30)\end{array}$ & $\begin{array}{c}2.21 \\
(1.02-5.20)\end{array}$ \\
\hline \multicolumn{8}{|l|}{ History of asthma } \\
\hline Yes & $\begin{array}{c}0.02 \\
(0.02-0.03)\end{array}$ & $\begin{array}{c}0.02 \\
(0.00-0.16)\end{array}$ & $\begin{array}{c}0.92 \\
(0.13-6.65)\end{array}$ & $\begin{array}{c}0.02 \\
(0.00-0.16)\end{array}$ & $\begin{array}{c}0.92 \\
(0.13-6.65)\end{array}$ & $\begin{array}{c}0.09 \\
(0.03-0.24)\end{array}$ & $\begin{array}{c}3.70 \\
(1.35-10.12)\end{array}$ \\
\hline No & $\begin{array}{c}0.12 \\
(0.11-0.13)\end{array}$ & $\begin{array}{c}0.18 \\
(0.09-0.36)\end{array}$ & $\begin{array}{c}1.48 \\
(0.73-2.97)\end{array}$ & $\begin{array}{c}0.13 \\
(0.6-0.30)\end{array}$ & $\begin{array}{c}1.11 \\
(0.49-2.48)\end{array}$ & $\begin{array}{c}0.20 \\
(0.10-0.38)\end{array}$ & $\begin{array}{c}1.66 \\
(0.86-3.22)\end{array}$ \\
\hline \multicolumn{8}{|c|}{ Severity of reaction } \\
\hline Mild & $\begin{array}{c}0.03 \\
(0.02-0.03)\end{array}$ & $0.0 \dagger \ddagger$ & $0 \ddagger$ & $\begin{array}{c}0.09 \\
(0.03-0.24)\end{array}$ & $\begin{array}{c}3.46 \\
(1.27-9.46)\end{array}$ & $\begin{array}{c}0.07 \\
(0.02-0.21)\end{array}$ & $\begin{array}{c}2.60 \\
(0.82-8.23)\end{array}$ \\
\hline Moderate & $\begin{array}{c}0.11 \\
(0.10-0.12)\end{array}$ & $\begin{array}{c}0.20 \\
(0.10-0.38)\end{array}$ & $\begin{array}{c}1.81 \\
(0.93-3.51)\end{array}$ & $\begin{array}{c}0.07 \\
(0.02-0.21)\end{array}$ & $\begin{array}{c}0.60 \\
(0.19-1.88)\end{array}$ & $\begin{array}{c}0.20 \\
(0.10-0.38)\end{array}$ & $\begin{array}{c}1.81 \\
(0.93-3.51)\end{array}$ \\
\hline Severe & $-\S$ & $-\S$ & $-\S$ & $-\S$ & $-\S$ & $-\S$ & $-\S$ \\
\hline \multicolumn{8}{|l|}{ Hospital centre } \\
\hline $\mathrm{MCH}$ & $\begin{array}{c}0.10 \\
(0.09-0.11)\end{array}$ & $\begin{array}{c}0.09 \\
(0.03-0.24)\end{array}$ & $\begin{array}{c}0.87 \\
(0.32-2.33)\end{array}$ & $\begin{array}{c}0.16 \\
(0.07-0.33)\end{array}$ & $\begin{array}{c}1.52 \\
(0.72-3.22)\end{array}$ & $\begin{array}{c}0.18 \\
(0.09-0.36)\end{array}$ & $\begin{array}{c}1.74 \\
(0.86-3.51)\end{array}$ \\
\hline $\mathrm{BCCH}$ & $\begin{array}{c}0.03 \\
(0.02-0.03)\end{array}$ & $\begin{array}{c}0.09 \\
(0.03-0.24)\end{array}$ & $\begin{array}{c}3.34 \\
(1.22-9.11)\end{array}$ & $0.0 \dagger \ddagger$ & $0.0 \ddagger$ & $\begin{array}{c}0.11 \\
(0.05-0.27)\end{array}$ & $\begin{array}{c}4.17 \\
(1.69-10.29)\end{array}$ \\
\hline $\begin{array}{l}\text { Note: } \mathrm{BCCH}=\text { British } \\
{ }^{*} \text { Calculated with rest } \\
\text { †Value is } 1.89 \mathrm{E}-11 . \\
\text { †Confidence interval } \\
\text { §Too few events - n }\end{array}$ & $\begin{array}{l}\text { dren's Hospital, } \\
\text { arence category. } \\
\text { ately large. }\end{array}$ & fidence interv & incidence rat & $\mathrm{MCH}=$ Montre & en's Hospital. & & \\
\hline
\end{tabular}

In subgroup analyses, we found that the IRR was higher during Halloween and Easter for children aged 6 years or more than for younger children, most probably because of decreased parental supervision. ${ }^{19}$ We also found that children recruited prospectively had a higher IRR during Easter for all 3 allergens, whereas for children recruited retrospectively there was a higher IRR during Halloween for unknown nuts and peanuts. The differences in holiday effect between the 2 groups are unlikely related to method of recruitment; our exposure, holiday time period, was indicated in the chart and is an objective measure (i.e., not subject to recruitment time). The observed differences are likely related to variation in sample size between our prospective (588 patients) and retrospective (802 patients) cohorts. Furthermore, patient sociodemographic and clinical characteristics did not differ between the 2 groups. Finally, we observed that the IRR was higher for unknown nuts and peanuts during Halloween among children recruited from the Montreal Children's Hospital, whereas the IRR was higher for unknown nuts and tree nuts during Easter for children recruited from British Columbia Children's Hospital, in Vancouver. This may reflect different food products and lifestyles between the 2 cities. ${ }^{20}$ 
Table 6: Characteristics of patients by recruitment method

\begin{tabular}{|c|c|c|c|}
\hline \multirow[b]{2}{*}{ Characteristic } & \multicolumn{3}{|c|}{ No. $(\%)$ of patients ${ }^{\star}$; method } \\
\hline & $\begin{array}{c}\text { Retrospective } \\
n=802\end{array}$ & $\begin{array}{c}\text { Prospective } \\
n=588\end{array}$ & $\begin{array}{c}\text { Total } \\
n=1390\end{array}$ \\
\hline \multicolumn{4}{|l|}{ Sex } \\
\hline Male & $497(62.0)$ & $367(62.4)$ & $864(62.2)$ \\
\hline Female & $305(38.0)$ & $221(37.6)$ & $526(37.8)$ \\
\hline $\begin{array}{l}\text { Age, median (IQR), } \\
\text { yr }\end{array}$ & $5.8(2.9-10.8)$ & $5.1(2.2-11.0)$ & $5.4(2.5-10.9)$ \\
\hline \multicolumn{4}{|l|}{ History of asthma } \\
\hline Yes & $137(17.1)$ & $94(16.0)$ & $231(16.6)$ \\
\hline No & $660(82.3)$ & $492(83.6)$ & $1152(82.9)$ \\
\hline $\begin{array}{l}\text { Information } \\
\text { missing }\end{array}$ & $\leq 5$ & $\leq 5$ & $7(0.5)$ \\
\hline \multicolumn{4}{|c|}{ Known peanut allergy } \\
\hline Yes & $316(39.4)$ & $213(36.2)$ & $529(38.1)$ \\
\hline No & $481(60.0)$ & $373(63.4)$ & $854(61.4)$ \\
\hline $\begin{array}{l}\text { Information } \\
\text { missing }\end{array}$ & $\leq 5$ & $\leq 5$ & $7(0.5)$ \\
\hline \multicolumn{4}{|c|}{ Known tree nut allergy } \\
\hline Yes & $155(19.3)$ & $87(14.8)$ & $242(17.4)$ \\
\hline No & $642(80.1)$ & $499(84.8)$ & $1141(82.1)$ \\
\hline $\begin{array}{l}\text { Information } \\
\text { missing }\end{array}$ & $\leq 5$ & $\leq 5$ & $7(0.5)$ \\
\hline
\end{tabular}

Our findings, which suggest higher risk of anaphylaxis induced by unknown nuts and peanuts during Halloween and Easter than during the rest of the year, differ from those of 2 previous, non-peer-reviewed reports. In 2015, a report from the Canadian Institute for Health Information documented higher rates of food-induced allergic reaction presentations to emergency departments in December, May, July and August than during other months. ${ }^{21} \mathrm{~A}$ survey conducted by the pharmaceutical company Mylan showed higher incidence rates of anaphylaxis, whether food-induced or latex-induced, in winter among 300 US children. ${ }^{22}$ However, in contrast to our study, these reports were not based on data collection prospectively and retrospectively from the emergency department and were not based on a consensus definition of anaphylaxis. Therefore, they are more prone to misclassification bias.

The labelling of common allergens is mandatory in Canada. The list of common allergens, which includes peanuts and tree nuts, is determined by Health Canada and encompasses allergens accounting for most food allergies in Canadians. ${ }^{23}$ Despite this, our findings suggest that educational tools to increase vigilance regarding the presence of potential allergens is required among children with food allergies, their families and lay people interacting with children who have food allergies. Newer strategies targeting intervals associated with high anaphylaxis risk are required. An example of such strategies are Food Allergy Canada's Shine a Teal Light campaign and the community Teal Pumpkin Project. ${ }^{24-28}$ Incorporating such strategies will contribute to making holidays safer and more inclusive for children with peanut and tree nut allergy.

\section{Limitations}

It is possible that patients with mild anaphylactic reactions or known allergy were treated outside of the emergency department and, thus, did not present to a hospital for additional treatment. However, a previous study by our group suggested that most cases meeting the criteria for anaphylaxis present to the emergency department. ${ }^{29}$ Another possible limitation is that we included only 6 academic hospitals, and community hospitals were not involved in this study. However, we believe our sample is representative of Canadian children as we included patients from centres throughout the country. Furthermore, we collected data both prospectively and retrospectively, but we do not believe the method of recruitment affected our findings substantially, as patient sociodemographic and clinical characteristics did not differ among the groups, and the dates and factors assessed (age, sex, severity of reaction) are not likely to be misclassified even in retrospective documentation. When we compared the average daily count of events between the week before and after each holiday, for all 3 culprits, no significant differences were found for any holiday, likely owing to the small samples. Hence, we cannot rule out the effect of seasonality and overcrowding of emergency departments. In addition, given the relatively small samples, we were not able to determine specific associations between peanut- or tree-nut-induced reactions and specific sociodemographic factors or comorbidities through a multivariable analysis. Instead, we used a Poisson regression model and performed a stratified analysis. Finally, we were not able to evaluate the effect of culture and diet on IRRs, given that data were not collected for these variables.

\section{Conclusion}

We found an increased risk of anaphylaxis induced by unknown nuts and peanuts during Halloween and Easter among Canadian children. We also found that the holiday period particularly affected older children. Educational programs and strategies increasing vigilance among families of children with peanut or tree nut allergy and among people interacting with them are required in order to render holidays safer for all Canadian children.

\section{References}

1. Clarke AE, Elliott SJ, St Pierre Y, et al. Temporal trends in prevalence of food allergy in Canada. J Allergy Clin Immunol Pract 2020;8:1428-30.e5.

2. Soller L, Ben-Shoshan M, Harrington DW, et al. Adjusting for nonresponse bias corrects overestimates of food allergy prevalence. J Allergy Clin Immunol Pract 2015;3:291-3.e2.

3. Ben-Shoshan M, Turnbull E, Clarke A. Food allergy: temporal trends and determinants. Curr Allergy Asthma Rep 2012;12:346-72.

4. Cohen N, Capua T, Pivko D, et al. Trends in the diagnosis and management of anaphylaxis in a tertiary care pediatric emergency department. Ann Allergy Asthma Immunol 2018;121:348-52. 
5. Sampson HA, Muñoz-Furlong A, Campbell RL, et al. Second symposium on the definition and management of anaphylaxis: summary report - Second National Institute of Allergy and Infectious Disease/Food Allergy and Anaphylaxis Network symposium. J Allergy Clin Immunol 2006;117:391-7.

6. Hochstadter E, Clarke A, De Schryver S, et al. Increasing visits for anaphylaxis and the benefits of early epinephrine administration: a 4-year study at a pediatric emergency department in Montreal, Canada. J Allergy Clin Immunol 2016; 137:1888-90.e4.

7. Lee $\mathrm{AY}$, Enarson $\mathrm{P}$, Clarke AE, et al. Anaphylaxis across two Canadian pediatric centers: evaluating management disparities. J Asthma Allergy 2016;10:1-7.

8. Ben-Shoshan M, Clarke AE. Anaphylaxis: past, present and future. Allergy 2011; 66:1-14.

9. Ben-Shoshan M, La Vieille S, Eisman H, et al. Anaphylaxis treated in a Canadian pediatric hospital: incidence, clinical characteristics, triggers, and management. J Allergy Clin Immunol 2013;132:739-41.e3.

10. Xu YS, Kastner M, Harada L, et al. Anaphylaxis-related deaths in Ontario: a retrospective review of cases from 1986 to 2011. Allergy Asthma Clin Immunol 2014;10:38.

11. Brown SG. Clinical features and severity grading of anaphylaxis. J Allergy Clin Immunol 2004;114:371-6.

12. Pumphrey RS. Lessons for management of anaphylaxis from a study of fatal reactions. Clin Exp Allergy 2000;30:1144-50.

13. Muraro A, Roberts G, Clark A, et al.; EAACl Task Force on Anaphylaxis in Children. The management of anaphylaxis in childhood: position paper of the European Academy of Allergology and Clinical Immunology. Allergy 2007;62: 857-71.

14. Gabrielli S, Clarke A, Morris J, et al. Evaluation of prehospital management in a Canadian emergency department anaphylaxis cohort. J Allergy Clin Immunol Pract 2019;7:2232-8.e3.

15. Katz SH, Weaver WW. Encyclopedia of food and culture. New York: Scribner; 2003.

16. Higgs S, Thomas J. Social influences on eating. Curr Opin Behav Sci 2016;9:1-6.

17. Shatenstein B, Ghadirian P. Influences on diet, health behaviours and their outcome in select ethnocultural and religious groups. Nutrition 1998;14:223-30.

18. Labelling requirements for confectionery, chocolate and snack food products. Government of Canada. Ottawa: Canadian Food Inspection Agency; modified 2019 Jan. 15. Available: https://inspection.gc.ca/food-label-requirements/ labelling/industry/confectionery-chocolate-and-snack-food-products/eng/139 2136343660/1392136466186? chap=0\#s1c2 (accessed 2020 Feb. 27)

19. Gabrielli S, Clarke A, Morris J, et al. Teenagers and those with severe reactions are more likely to use their epinephrine autoinjector in cases of anaphylaxis in Canada. J Allergy Clin Immunol Pract 2019;7:1073-5.e3.

20. Religion (108), immigrant status and period of immigration (11), age groups (10) and sex (3) for the population in private households of Canada, provinces, territories, census metropolitan areas and census agglomerations, 2011 National Household Survey. Ottawa: Statistics Canada; 2013. Cat no 99-010-X2011032.

21. Anaphylaxis and allergy in the emergency department [pamphlet]. Ottawa: Canadian Institute for Health Information; 2015

22. Incidence of anaphylaxis during winter events highlights importance of adding a preparedness checklist to holiday planning [press release]. Canonsburg (PA): Mylan N.V.; 2012 Dec. 18. Available: http://newsroom.mylan.com/press -releases?item=123064 (accessed 2020 Feb. 27).

23. Food allergen labelling. Ottawa: Health Canada; modified 2016 Dec. 14. Available: www.canada.ca/en/health-canada/services/food-nutrition/food -labelling/allergen-labelling.html (accessed 2019 Nov. 25).

24. Ben-Shoshan M, Sheth S, Harrington D, et al. Effect of precautionary statements on the purchasing practices of Canadians directly and indirectly affected by food allergies. J Allergy Clin Immunol 2012;129:1401-4.

25. \#ShineATealLight on food allergy this Halloween. Toronto: Food Allergy Canada. Available: www.foodallergycanada.ca/campaign/shineateallight-on-food-allergy -this-halloween/shine-a-teal-light-on-food-allergy-this-halloween/ (accessed 2019 Nov. 25).

26. Teal Pumpkin Project ${ }^{\circledR}$. McLean (VA): Food Allergy Research \& Education. Available: www.foodallergy.org/education-awareness/teal-pumpkin-project (accessed 2019 Nov. 25)

27. Allergens and gluten sources labelling. Ottawa: Health Canada; modified 2019 May 6. Available: www.canada.ca/en/health-canada/services/food-allergies -intolerances/avoiding-allergens-food/allergen-labelling.html (accessed 2019 Nov. 25).

28. Priority food allergens. Toronto: Food Allergy Canada. Available: https:// foodallergycanada.ca/food-allergy-basics/food-allergies-101/what-are-food -allergies/priority-food-allergens/ (accessed 2019 Nov. 25).

29. O'Keefe A, Clarke A, St Pierre Y, et al. The risk of recurrent anaphylaxis. J Pediatr 2017;180:217-21.
Competing interests: Judy Morris received a grant from AllerGen Canada (Allergy, Genes and Environment Network), a Networks of Centres of Excellence research network, to allow the data for the project to be collected at her university hospital. AllerGen Canada is a not-for-profit network based at McMaster University. Julia Upton received advisor fees from Food Allergy Canada, ALK-Abelló, Kaléo and Bausch Health; research grant support from the Toronto SickKids Food Allergy and Anaphylaxis fund, DBV Technologies, Regeneron, the Canadian Institutes of Health Research and ALK-Abelló; and an in-kind contribution from Novartis, outside the submitted work. No other competing interests were declared.

This article has been peer reviewed.

Affiliations: Division of Allergy and Clinical Immunology (Leung, Gabrielli, Ben-Shoshan), Department of Pediatrics, Montreal Children's Hospital, McGill University Health Centre, Montréal, Que.; Division of Rheumatology (Clarke, Shand), Department of Medicine, Cumming School of Medicine, University of Calgary, Calgary, Alta.; Department of Emergency Medicine (Morris), Hôpital Sacré-Cœur;
Division of Pediatric Emergency Medicine (Gravel), Department of Pediatrics, Centre hospitalier universitaire Sainte-Justine, Montréal, Que.; Division of Pediatric Emergency Medicine (Lim), Department of Pediatrics, Children's Hospital at London Health Sciences Centre, London, Ont.; Divisions of Allergy and Immunology (Chan) and Emergency Medicine (Goldman, Enarson), Department of Pediatrics, BC Children's Hospital, University of British Columbia, Vancouver, BC; Department of Pediatrics (O'Keefe), Faculty of Medicine, Memorial University, St. John's, NL; Food Allergy Canada (Gerdts), Toronto, Ont.; Division of Clinical Immunology \& Allergy (Chu), Department of Medicine, and Department of Health Research Methods, Evidence, and Impact (Chu), McMaster University, Hamilton, Ont.; Division of Immunology and Allergy (Upton), Department of Pediatrics, The Hospital for Sick Children, University of Toronto, Toronto, Ont.; Centre for Outcomes Research and Evaluation (Zhang), Research Institute of McGill University Health Centre, Montréal, Que.

Contributors: Greg Shand conceived the work, and Moshe Ben-Shoshan, Greg Shand,
Ann Clarke and Sofianne Gabrielli designed it. Mélanie Leung, Sofianne Gabrielli, Judy Morris, Jocelyn Gravel, Rodrick Lim, Edmond Chan, Ran Goldman, Andrew O'Keefe, Greg Shand and Paul Enarson contributed to data acquisition. Mélanie Leung, Xun Zhang, Greg Shand and Moshe Ben-Shoshan analyzed the data. Mélanie Leung drafted the manuscript under the supervision of Moshe Ben-Shoshan. All of the authors contributed to data interpretation, revised the manuscript critically for important intellectual content, approved the final version to be published and agreed to be accountable for all aspects of the work.

Funding: This study was supported by operating grant GEN 10-203 from AllerGen Canada.

Disclaimer: The sponsor had no role in the design, conduct, analysis or interpretation, or the decision to submit the manuscript for publication.

Data sharing: The data are not available to other investigators.

Accepted: May 15, 2020

Correspondence to: Melanie Leung, melanie.leung@mail.mcgill.ca 